\title{
Monitoring immunotherapy outcomes with circulating tumor DNA
}

\author{
Sarah B Goldberg ${ }^{1}$ \& Abhijit A Patel*,2 \\ ${ }^{1}$ Department of Medicine (Medical Oncology), Yale School of Medicine, Yale University, New Haven, CT, USA \\ ${ }^{2}$ Department of Therapeutic Radiology, Yale School of Medicine, Yale University, New Haven, CT, USA \\ *Author for correspondence: abhijit.patel@yale.edu
}

'While results of these preliminary studies appear to be compelling, additional validation studies in larger cohorts are needed to support the routine clinical use of ctDNA as a biomarker of immunotherapy response."

First draft submitted: 19 June 2018; Accepted for publication: 11 July 2018; Published online: 6 September 2018

Keywords: cell-free DNA • circulating tumor DNA • ctDNA • immune checkpoint inhibitor • immunotherapy • lung cancer

\section{Immunotherapy in non-small-cell lung cancer}

Over the past several years, PD-1 and PD-L1 inhibitors have transformed the way in which patients with lung cancer are treated. Based on multiple clinical trials demonstrating an improvement in survival with these agents, both alone and in combination with chemotherapy, immunotherapies are now a standard aspect of the management of patients with advanced non-small-cell lung cancer (NSCLC) [1,2]. Incredibly, long-term survival is now possible in more patients with metastatic NSCLC than ever before, with 5-year survival rates of approximately $15 \%$ with the PD-1 inhibitor nivolumab [3]. However, in all-comers with advanced NSCLC, response rates to single-agent PD-1 axis inhibitors are only around 20\% [4,5], indicating that the majority of patients will not benefit from this class of agents.

There has been intense investigation directed at identifying biomarkers that may predict which patients with lung cancer are most likely to respond to immunotherapy, both initially and in the long-term. Tumor PD-L1 expression as assessed by immunohistochemistry has emerged as the standard assay used to predict therapeutic benefit. However, this biomarker has limited sensitivity and specificity, resulting in many patients being inappropriately offered or denied immune checkpoint inhibitor therapy [4].

A further complicating aspect of evaluating benefit from immunotherapy is that radiographic responses can be atypical: patients can have transient tumor growth or appearance of new lesions prior to tumor shrinkage (known as 'pseudo-progression') and in some cases response can take many months to manifest. For the oncologist, the challenges in interpreting radiographic response can make it difficult to decide whether or not to continue a patient on immunotherapy. The current biomarker landscape does not assist with this sort of decision-making and judgement must be made based on clinical features and radiographic findings.

\section{Circulating tumor DNA as a biomarker of therapy response}

Circulating tumor DNA (ctDNA) is already being used clinically in the management of patients with NSCLC, particularly for noninvasive genotyping of somatic tumor mutations to guide selection of therapy. However, many other applications of ctDNA are being actively investigated, including its potential use as a biomarker of treatment response. Because tumor-specific mutations can be used to identify ctDNA, it is expected to have greater cancer specificity than most serum protein biomarkers. Moreover, because ctDNA is shed from dying cancer cells and is

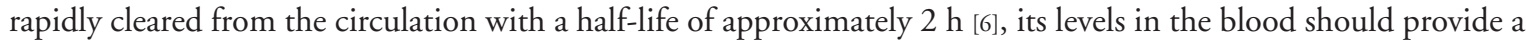
real-time assessment of active cell death. In contrast, most serum protein markers are secreted from living cells and are reflective of tumor burden. For example, a bulky pancreatic tumor with very few dying cells would be predicted to secrete abundant CA-19-9, but would shed little KRAS-mutant ctDNA. Accordingly, ctDNA levels would be 
expected to change more rapidly in response to successful therapy than would protein marker levels, because the rate of tumor cell death is likely to change more rapidly than the total number of tumor cells. Such rapid ctDNA kinetics have indeed been observed following treatment in several studies [6-10]: ctDNA levels can initially spike as large numbers of tumor cells are killed, and can then rapidly drop as the rate of cell death declines. These features of ctDNA make it an attractive marker of treatment response, especially in clinical settings where protein biomarkers perform poorly or are nonexistent, and radiographic findings are difficult to interpret.

\section{Using ctDNA to monitor response \& predict patient outcomes with immunotherapy}

There is growing evidence that tracking changes in ctDNA levels in serial samples during immunotherapy can enable assessment of therapeutic benefit. Thus far, the use of ctDNA to monitor treatment response during immunotherapy has been studied most extensively in patients with advanced melanoma. The reason for this is likely twofold: (1) Immune checkpoint inhibitors have a longer history of use in melanoma than in NSCLC, so banked specimens may be more readily available and (2) BRAF and NRAS hotspot mutations frequently found in melanoma can be easily measured using cheaper, targeted assays such as digital PCR (rather than broad-panel next-generation sequencing). In one of the earliest such studies, ctDNA levels were tracked in four patients with melanoma; three of these patients showed increasing ctDNA levels in conjunction with progressive disease, whereas one patient had early clinical progression, but then ctDNA levels became undetectable 3 weeks prior to clinical tumor regression [11]. Another study of 76 patients with melanoma treated with immunotherapy found that patients with undetectable ctDNA at baseline and those whose ctDNA became undetectable within 12 weeks of starting treatment had significantly better progression-free survival (PFS) and overall survival (OS) than patients whose ctDNA was elevated at baseline and remained such during treatment [12]. The same group later published another study of patients with melanoma in which they demonstrated that ctDNA profiles could differentiate pseudoprogression from true disease progression in the setting of anti-PD-1 therapy [13]. Yet another study found that tracking ctDNA levels at baseline and at 8 weeks post-treatment could predict radiographic response, PFS and OS in 10 patients treated with PD-1 inhibitors for either uveal melanoma, colorectal cancer, or NSCLC [14]. Finally, our group published a study focusing exclusively on patients with NSCLC receiving PD-1 axis therapy, quantifying ctDNA via deep sequencing. Among a cohort of 28 patients with detectable ctDNA pretreatment, a decrease in ctDNA levels to less than $50 \%$ of baseline was associated with significantly superior PFS, OS and longer duration on treatment [15]. This study also showed that such a 'ctDNA response' correlated strongly with radiographic response and could be detected at a median of 24.5 days post-treatment, compared with a median of 72.5 days for radiographic response. Together, these studies provide compelling evidence that in retrospective cohorts of multiple disease types receiving a variety of immune checkpoint inhibitors, monitoring of ctDNA levels appears to enable rapid assessment of treatment response and prediction of clinical outcomes.

\section{Caveats \& future perspective}

There are several limitations that have become apparent in studies investigating ctDNA as a marker of immunotherapy efficacy. First, it will not be possible to track ctDNA in all patients. Some patients will have tumors that do not shed sufficient DNA to be detected, and others will have tumors that harbor mutations which are not covered by the assay. Second, the approach of measuring quantitative changes in ctDNA requires testing of serial samples during therapy and therefore cannot be used to predict efficacy prior to starting treatment. Broad-panel sequencing of cell-free DNA to noninvasively assess tumor mutation burden is showing promise as an alternative approach to predicting immunotherapy efficacy before starting treatment [16-18]. Third, a spike in ctDNA level can sometimes be observed soon after the start of therapy, likely due to tumor cell death, but this could be mistaken as being indicative of disease progression. Such an early spike has been observed in several patients during the first approximately 1 week following T-cell transfer therapy in patients with melanoma and was associated with response to treatment [9]. The timing and magnitude of such ctDNA spikes will need to be further characterized for different cancer types and different immunotherapies to better understand how to interpret post-treatment ctDNA trends. Finally, while results of these preliminary studies appear to be compelling, additional validation studies in larger cohorts are needed to support the routine clinical use of ctDNA as a biomarker of immunotherapy response. However, the striking trends observed thus far in multiple independent studies suggest that ctDNA monitoring will likely find utility in conjunction with pretreatment predictive biomarkers and standard radiographic response assessment to guide the treatment of patients with immunotherapy. 


\section{Financial \& competing interests disclosure}

AA Patel has received a research grant from AstraZeneca and has served in advisory roles for Novartis and NuGen Technologies. $\mathrm{He}$ is also an inventor on pending and awarded patents covering ctDNA assay technologies. SB Goldberg has received a research grant from AstraZeneca and has served in advisory roles for AstraZeneca, Boehringer Ingelheim, Bristol-Myers Squibb and Lilly. This work was supported in part by grants from the National Cancer Institute (R01-CA197486), the Yale SPORE in Lung Cancer (P50-CA196530), the Yale Cancer Center (P30-CA016359), the LUNGevity Foundation, the Honorable Tina Brozman Foundation. The authors have no other relevant affiliations or financial involvement with any organization or entity with a financial interest in or financial conflict with the subject matter or materials discussed in the manuscript apart from those disclosed.

No writing assistance was utilized in the production of this manuscript.

\section{References}

1. Reck M, Rodriguez-Abreu D, Robinson AG et al. Pembrolizumab versus chemotherapy for PD-L1-positive non-small-cell lung cancer. N. Engl. J. Med. 375(19), 1823-1833 (2016).

2. Gandhi L, Rodriguez-Abreu D, Gadgeel S et al. Pembrolizumab plus chemotherapy in metastatic non-small-cell lung cancer. N. Engl. J. Med. 378(22), 2078-2092 (2018).

3. Gettinger S, Horn L, Jackman D et al. Five-year follow-up of nivolumab in previously treated advanced non-small-cell lung cancer: results from the CA209-003 Study. J. Clin. Oncol. 36(17), 1675-1684 (2018).

4. Garon EB, Rizvi NA, Hui R et al. Pembrolizumab for the treatment of non-small-cell lung cancer. N. Engl. J. Med. 372(21), 2018-2028 (2015).

5. Borghaei H, Brahmer J. Nivolumab in nonsquamous non-small-cell lung cancer. N. Engl. J. Med. 374(5), 493-494 (2016).

6. Diehl F, Schmidt K, Choti MA et al. Circulating mutant DNA to assess tumor dynamics. Nat. Med. 14(9), 985-990 (2008).

7. Dawson SJ, Tsui DW, Murtaza M et al. Analysis of circulating tumor DNA to monitor metastatic breast cancer. N. Engl. J. Med. 368(13), 1199-1209 (2013).

8. Pereira E, Camacho-Vanegas $\mathrm{O}$, Anand $\mathrm{S}$ et al. Personalized circulating tumor dna biomarkers dynamically predict treatment response and survival in gynecologic cancers. PLoS ONE 10(12), e0145754 (2015).

9. Xi L, Pham TH, Payabyab EC, Sherry RM, Rosenberg SA, Raffeld M. Circulating tumor dna as an early indicator of response to t-cell transfer immunotherapy in metastatic melanoma. Clin. Cancer Res. 22(22), 5480-5486 (2016).

10. Girotti MR, Gremel G, Lee R et al. Application of sequencing, liquid biopsies, and patient-derived xenografts for personalized medicine in melanoma. Cancer Discov.6(3), 286-299 (2016).

11. Lipson EJ, Velculescu VE, Pritchard TS et al. Circulating tumor DNA analysis as a real-time method for monitoring tumor burden in melanoma patients undergoing treatment with immune checkpoint blockade. J. Immunother. Cancer 2(1), 42 (2014).

12. Lee JH, Long GV, Boyd S et al. Circulating tumour DNA predicts response to anti-PD1 antibodies in metastatic melanoma. Ann. Oncol. 28(5), 1130-1136 (2017).

13. Lee JH, Long GV, Menzies AM et al. Association between circulating tumor dna and pseudoprogression in patients with metastatic melanoma treated with anti-programmed cell death 1 antibodies. JAMA Oncol., 4(5), 717-721 (2018).

14. Cabel L, Riva F, Servois V et al. Circulating tumor DNA changes for early monitoring of anti-PD1 immunotherapy: a proof-of-concept study. Ann. Oncol. 28(8), 1996-2001 (2017).

15. Goldberg SB, Narayan A, Kole AJ et al. Early assessment of lung cancer immunotherapy response via circulating tumor DNA. Clin. Cancer Res. 24(8), 1872-1880 (2018).

16. Khagi Y, Goodman AM, Daniels GA et al. Hypermutated circulating tumor dna: correlation with response to checkpoint inhibitor-based immunotherapy. Clin. Cancer Res. 23(19), 5729-5736 (2017).

17. Fabrizio D, Malboeuf C, Lieber D et al. Analytic validation of a next generation sequencing assay to identify tumor mutational burden from blood (bTMB) to support investigation of an anti-PD-L1 agent, atezolizumab, in a first line non-small cell lung cancer trial (BFAST). Ann. Oncol. 28, (2017).

18. Gandara DR, Kowanetz M, Mok TSK et al. Blood-based biomarkers for cancer immunotherapy: tumor mutational burden in blood (bTMB) is associated with improved atezolizumab (atezo) efficacy in 2L+NSCLC (POPLAR and OAK). Ann. Oncol. 28, (2017). 
